\title{
Effects of small platform catheter-based left ventricular assist device support on regional myocardial signal transduction
}

\author{
Keshava Rajagopal, MD, PhD, Progyaparamita Saha, PhD, Isa Mohammed, BS, \\ Pablo G. Sanchez, MD, PhD, Tieluo Li, MD, Zhongjun J. Wu, PhD, and Bartley P. Griffith, MD
}

\section{ABSTRACT}

Objectives: Left ventricular (LV) assist device (LVAD) support reduces pathological loading. However, load-induced adaptive responses may be suppressed. Pathological loading dysregulates cardiac $G$ protein-coupled receptor (GPCR) signaling. Signaling through $G$ proteins is deleterious, whereas beta $(\beta)$-arrestin-mediated signaling is cardioprotective. We examined the effects of pathological LV loading/LV dysfunction and treatment via LVAD, on $\beta$-arrestin-mediated signaling, and genetic networks downstream of load.

Methods: An ovine myocardial infarction (MI) model was used. Sheep underwent sham thoracotomy $(n=3)$, mid-left anterior descending coronary artery ligation to produce MI $(\mathrm{n}=3)$, or MI with placement of a small-platform catheter-based LVAD ( $\mathrm{n}=3$ ). LVAD support was continued for 2 weeks. Animals were maintained for a total of 12 weeks. Myocardial specimens were harvested and analyzed.

Results: MI induced $\beta$-arrestin activation. Increased interactions between epidermal growth factor receptor and $\beta$-arrestins were observed. LVAD support inhibited these responses to MI $(P<.05)$. LVAD support inhibited the activation of cardioprotective signaling effectors Akt $(P<.05)$, and, to a lesser extent, extracellular regulated kinase $1 / 2$ ( $P$ not significant); however, MI resulted in regional activation of load-induced GPCR signaling via G proteins, as assessed by the induction of atrial natriuretic peptide mRNA expression in the MI-adjacent zone relative to the MI-remote zone $(P<.05)$. MI-adjacent zone atrial natriuretic peptide expression was renormalized with LVAD support.

Conclusions: LVAD support inhibited cardioprotective $\beta$-arrestin-mediated signaling. However, net benefits of normalization of load-induced GPCR signaling were observed in the MI-adjacent zone. These findings may have implications for the optimal extent and duration of unloading, and for the development of adjunctive medical therapies. (J Thorac Cardiovasc Surg 2015;150:1332-41)

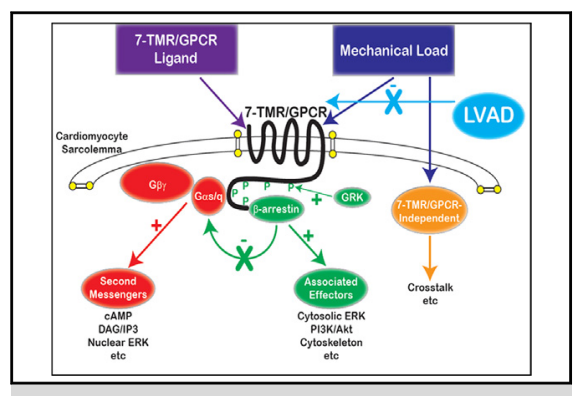

Cartoon diagram of the hypothesized effects of mechanical loading, with or without LVAD support, on cardiac 7-TMR/GPCR signal transduction. Mechanical load is depicted as acting like a ligand in facilitating cellsurface receptor (GPCR) activation. This activates both G protein-mediated signaling and $\beta$-arrestin-mediated signaling, the latter of which also suppresses $G$ protein coupling to the receptor (desensitization). As discussed in the text, chronic $\mathrm{G}$ protein and second messenger activation is cytotoxic, whereas several lines of evidence demonstrate that $\beta$-arrestin-mediated signaling is cytoprotective. As a result, it is suggested that load reduction might not selectively reduce $G$ protein activation, and also may reduce $\beta$-arrestin activation. The effects of the extent of loading/unloading and timing of LVAD support on differential pathway activity are unclear.

\section{Central Message}

Left ventricular assist device support inhibits pathologic responses to mechanical loading, but also can inhibit adaptive responses.

\section{Perspective}

Pathologic cardiac loading conditions occur following myocardial infarction, but load induces both directly pathological and adaptive signals. Thus, mechanical circulatory support may inhibit both types of signals. We show that nonselective suppression of both deleterious and beneficial signals occurs in response to left ventricular assist device support in a large-animal myocardial infarction model.

See Editorial Commentary page 1342.
From the Division of Cardiac Surgery, Department of Surgery, University of Maryland School of Medicine and Medical Center, Baltimore, Md.

This work was funded by the Norman E. Shumway Career Development Award of the International Society for Heart and Lung Transplantation (to K.R.) and National Institutes of Health, National Heart, Lung, and Blood Institute Grants 1R01 HL 124170 (to Z.J.W.) and 1R01 HL 118372 (to B.P.G.).

Dr Rajagopal's current affiliation is the Department of Cardiovascular Surgery and Center for Advanced Heart Failure, University of Texas-Houston, Memorial Hermann Hospital-Texas Medical Center, Houston, Tex. Dr Wu's current affiliation is the Department of Cardiovascular and Thoracic Surgery, University of Louisville School of Medicine, Louisville, Ky.
Read at the 95th Annual Meeting of The American Association for Thoracic Surgery, Seattle, Washington, April 25-29, 2015.

Received for publication May 4, 2015; revisions received July 20, 2015; accepted for publication Aug 8, 2015; available ahead of print Sept 19, 2015.

Address for reprints: Keshava Rajagopal, MD, PhD, Assistant Professor, Department of Cardiovascular Surgery, Cardiac Surgeon, Center for Advanced Heart Failure, University of Texas-Houston, Memorial Hermann Hospital-Texas Medical Center, 6400 Fannin St, Ste 2350, Houston, TX 77030 (E-mail: Keshava.Rajagopal@uth. tmc.edu).

$0022-5223 / \$ 36.00$

Copyright (c) 2015 by The American Association for Thoracic Surgery

http://dx.doi.org/10.1016/j.jtcvs.2015.08.035 


$$
\begin{aligned}
& \text { Abbreviations and Acronyms } \\
& \begin{aligned}
7-\mathrm{TMR} & =\text { 7-transmembrane receptor } \\
\mathrm{Ab} & =\text { Antibody } \\
\mathrm{AR} & =\text { Adrenergic receptor } \\
\mathrm{AT}_{1} \mathrm{R} & =\text { Angiotensin II type } 1 \text { receptor } \\
\mathrm{ECL} & =\text { Enhanced chemiluminescence } \\
\mathrm{EGFR} & =\text { Epidermal growth factor receptor } \\
\mathrm{ERK} & =\text { Extracellular regulated kinase } \\
\mathrm{ETR} & =\text { Endothelin A/B receptor } \\
\mathrm{GPCR} & =\text { G protein-coupled receptor } \\
\mathrm{GRK} & =\text { G protein-coupled receptor kinase } \\
\mathrm{HF} & =\text { Heart failure } \\
\mathrm{IPA} & =\text { Ingenuity Pathway Analysis } \\
\mathrm{LV} & =\text { Left ventricle/ventricular } \\
\mathrm{LVAD} & =\text { Left ventricular assist device } \\
\mathrm{MCS} & =\text { Mechanical circulatory support } \\
\mathrm{MI} & =\text { Myocardial infarction }
\end{aligned}
\end{aligned}
$$

The mortality and morbidity of acute myocardial infarction (MI) have been vastly improved with coronary arterial revascularization; however, many patients present or are treated in a delayed fashion. Consequently, left ventricular (LV) systolic dysfunction frequently complicates proximal coronary artery occlusion with large-territory MI. It is estimated that $\sim 30 \%$ of MIs result in heart failure (HF), ${ }^{1}$ and that $5 \%$ to $8 \%$ of MIs result in overt cardiogenic shock. ${ }^{2}$

Mechanical circulatory support (MCS) is now well validated as a therapy for both chronic and acute LV dysfunction when HF is present. ${ }^{3,4}$ However, it is less clear whether LV MCS at or around the time of MI actually attenuates pathological LV remodeling and reduces the extent/severity of $L V$ dysfunction. To address this question, we previously reported the development of a large-animal model of MI, and investigated the effects of short-term MCS via the Abiomed Impella smallplatform LV assist device (LVAD) on subsequent regional and global pathologic remodeling and systolic function. ${ }^{5}$ LVAD support for 2 weeks following MI resulted in reduced peri-infarct zone diastolic strains assessed at 12 weeks postMI, with improved (increased) peri-infarct zone systolic strains and LV ejection fraction. Another group has reported similar findings using ventricular restraint therapy. ${ }^{6}$

Regional myocardial and global LV contractility are consequences of myofilament function. ${ }^{7-9}$ In turn, myofilament physiological function is directly controlled by calcium handling within the cardiomyocyte. Consistent with these concepts, we found that deranged calcium handling in the MI-adjacent zone cardiomyocytes was substantially improved by LV MCS. However, the mechanisms-cell surface receptors and coupled intracellular signaling pathways - that link cardiomyocyte mechanical loading conditions to alterations in calcium handling, and how MCS in turn alters mechanotransduction, remain to be elucidated. The purpose of the present study was to investigate specific intracellular signaling pathways, outlined below, that are increasingly appreciated to play important roles in cardioprotective responses to cellular stresses such as mechanical loading.

Seven-transmembrane receptors (7-TMRs), also termed $\mathrm{G}$ protein-coupled receptors (GPCRs), are generally considered the most important receptors for regulating cardiac and vascular function ${ }^{10} ; 7$-TMR/GPCR pathways are the targets for the overwhelming majority of cardiovascular drug therapies. ${ }^{11}$ Numerous lines of evidence suggest that GPCRs, particularly G $\alpha$-coupled receptors, are critically involved in mechanotransduction and may even serve as direct mechanosensors. ${ }^{12-14}$

Canonical GPCR signaling is mediated by G protein activation, resulting in coupled second messenger molecule activation. This conventional pathway is antagonized by GPCR cytoplasmic tail phosphorylation, which is catalyzed by GPCR kinases (GRKs), of which 6 family members exist. $\beta$-arrestins (of which there are 2 types, $\beta$-arrestin 1 and $\beta$-arrestin2) bind to the tails, preventing further $\mathrm{G}$ protein coupling; this process is termed desensitization. ${ }^{15}$ However, $\beta$-arrestins not only antagonize $\mathrm{G}$ protein activation, but also couple GPCRs to downstream effector molecules. ${ }^{16}$ These effectors are distinct from those that the paired G protein/ second messengers stimulate. In the heart, $\beta$-arrestin signaling appears to enhance both cardiomyocyte function ${ }^{17}$ and survival, ${ }^{18}$ whereas $\mathrm{G}$ protein-mediated signaling enhances cardiomyocyte function acutely at the expense of long-term function and survival. LVAD support has been shown to inhibit chronic GPCR activation, specifically of $\beta$-adrenergic receptors (ARs) ${ }^{19,20}$; however, whether $\beta$-arrestin-mediated signaling is affected by this is unclear. The principal aim of the present study was to investigate the effects of MI and subsequent short-term LV MCS on $\beta$-arrestin-mediated signal transduction.

\section{METHODS}

\section{Large-Animal Model of MI, LV Dysfunction, and LVAD Support}

This model has been described in detail previously. ${ }^{5}$ In brief, adult Dorsett hybrid sheep (mean weight, $53.2 \pm 0.9 \mathrm{~kg}$ ) underwent sham thoracotomy, creation of large-territory MI by permanent ligation of the mid-left anterior descending coronary artery, or creation of MI followed by implantation of the Impella 5.0 small-platform catheter-based LVAD (Abiomed, Danvers, Mass) at the same setting (2-3 hours after MI). Pilot studies previously demonstrated that this results in infarction of $25 \%$ of the LV mass. Sonomicrometry crystal arrays were implanted subepicardially in all animals, to determine regional LV strains and global LV dimensions. In animals assigned to LVAD implantation, the pump speed was set between 20,000 and $24,000 \mathrm{rpm}$ to achieve $\sim 50 \%$ unloading of the total cardiac output. Support was continued for a 2-week period, and the animals were followed for an additional 10 weeks. All animals were monitored for 12 weeks after MI creation, at which point they were 
sacrificed and their hearts were harvested. All animals were treated in compliance with the National Institutes of Health's Guide for the Care and Use of Laboratory Animals (publication 85-23, revised 1996). All surgical procedures and postoperative care were approved by the Institutional Animal Care and Use Committee of the University of Maryland-Baltimore.

For the purpose of the present study, 3 animals in each group (control, MI alone, or MI + LVAD) had tissue samples available for analyses.

\section{Lysate Preparation and Immunoblotting}

At the time of termination, regional myocardium in the MI-adjacent zones (defined as $\leq 2 \mathrm{~cm}$ from the infarct border) and the MI-remote zones were harvested, snap-frozen in liquid nitrogen, and stored at $-80^{\circ} \mathrm{C}$ for future analysis. Lysates from cytosolic and membrane fractions were prepared using a cellular fraction and lysis kit (Pierce Thermo Scientific, Waltham, Mass). To determine phosphoprotein and total protein expression levels, cardiac lysates were subjected to gel electrophoresis using a $4 \%$ to $15 \%$ Tris- $\mathrm{HCl}$ Ready Gel (Bio-Rad, Hercules, Cal) and prepared using a cell fractionation kit (Pierce Thermo Scientific) with necessary modifications. Gels were transferred to membranes, and immunoblotting was performed. Analyses were performed on the following proteins, using antibodies (Abs) directed against phospho-Akt (T-308), total Akt, phospho-ERK1/2, total ERK1/2, $\beta$-arrestin1/2, and epidermal growth factor receptor (EGFR) (all primary Abs from Cell Signaling Technology, Danvers, Mass). Immunoreactivity was detected using enhanced chemiluminescence (ECL) (Hyglo; Denville Scientific, Metuchen, NJ) and photographic film (Hyblot CL; Denville Scientific). Densitometric quantification of the digitized immunoreactive bands was performed using UN-SCAN-IT Gel 5.1 software (Silk Scientific, Orem, Utah). Immunoblotting for the housekeeping protein glyceraldehyde-3phosphate dehydrogenase (1:2000 dilution; Santa Cruz Biotechnology, Santa Cruz, Cal) was used to confirm equal loading conditions for cytosolic protein expression.

Immunoprecipitation reactions were performed with a Pierce Crosslink Immunoprecipitation Kit (Pierce Thermo Scientific) following the manufacturer's protocol. Myocardial tissue cytosolic extracts were incubated with either anti- $\beta$-arrestin1/2 or anti-EGFR $\mathrm{Ab}$ at 1:50 dilution (Cell Signaling Technology and Santa Cruz Biotechnology, respectively) overnight at $4^{\circ} \mathrm{C}$, for assessment of $\beta$-arrestin-ubiquitin and $\beta$-arrestin-EGFR binding, respectively. Ab-bound immune complexes were retrieved as immunoprecipitates with a disuccinimidyl suberate-crosslinked protein A/G-agarose column. Immunoprecipitates were prepared in reducing sample buffer and subjected to gel electrophoresis. Gels were transferred to membranes and then immunoblotted with anti-ubiquitin $\mathrm{Ab}$ (Cell Signaling Technology) at 1:500 dilution for $\beta$-arrestin-ubiquitin interaction assessment, or with anti- $\beta$-arrestin 1/2 at 1:500 dilution for EGFR- $\beta$ arrestin interaction assessment. ECL was captured on photographic film, and densitometry was used to quantify band intensity.

\section{RNA Isolation and Sequencing}

RNA was isolated from $30 \mathrm{mg}$ of snap-frozen heart tissues using a Qiagen Fibrous Tissue Kit (Qiagen, Hilden, Germany) with several modifications. RNA was eluted with $25 \mu \mathrm{L}$ of RNAse-free water, and each sample was kept in a $-80^{\circ} \mathrm{C}$ freezer after aliquoting. The RNA integrity numbers of the extracted RNAs and their concentration $(\mathrm{ng} / \mu \mathrm{L})$ were determined by on-chip electrophoresis using an Agilent RNA Nano 6000 Lab Chip Kit and BioAnalyzer 2100 Expert (Agilent Technologies, Santa Clara, Calif).

RNA sequencing studies were performed at the University of Maryland-Baltimore RNA sequencing facility. Illumina RNAseq libraries were prepared with the TruSeq RNA Sample Prep kit (Illumina, San Diego, Calif) in accordance with the manufacturer's protocol. Adapters containing 6 nucleotide indices were ligated to the cDNA. cDNA was purified between enzymatic reactions, and the size selection of the library was performed

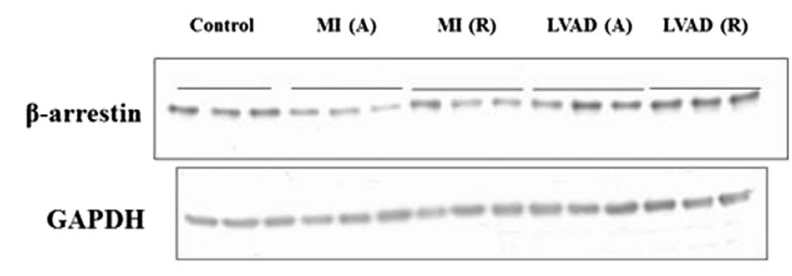

Total beta arrestin normalized by GAPDH
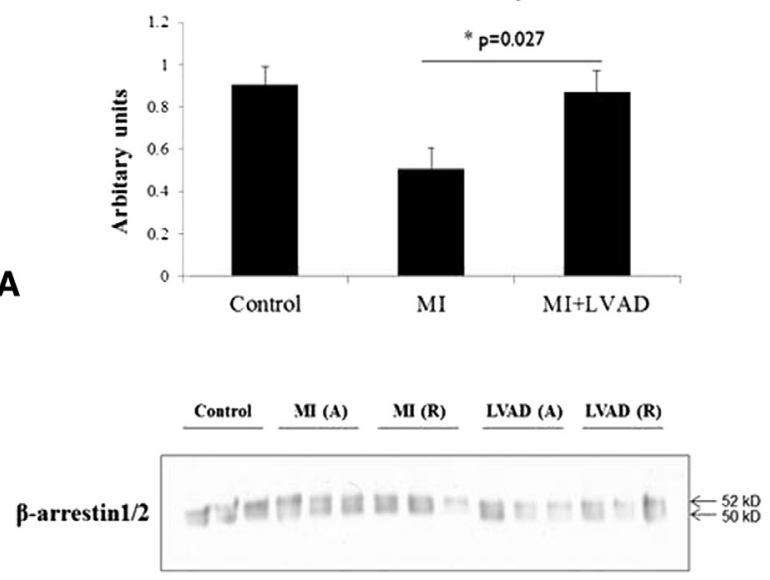

B

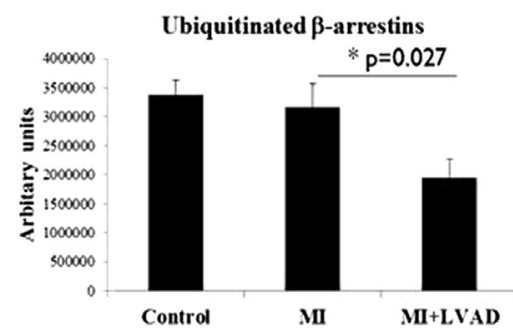

FIGURE 1. Effects of MI and LVAD support on $\beta$-arrestin levels and ubiquitination. A, adjacent; $\mathrm{R}$, remote. A, MI reduces $\beta$-arrestin $1 / 2$ levels, consistent with increased $\beta$-arrestin-mediated signaling. LVAD support restores $\beta$-arrestin $1 / 2$ levels, consistent with relative suppression of $\beta$-arrestin-mediated signaling. Immunoblotting was performed as described in Methods. Both $\beta$-arrestins present as a single band because whole-cell lysate samples were run for a shorter duration than immunoprecipitate samples (eg, panel B and Figure 2). B, LVAD support decreases levels of ubiquitinated $\beta$-arrestins, consistent with reduced GPCR internalization and $\beta$-arrestin-mediated signal transduction. Immunoprecipitations and immunoblotting were performed as described in Methods. MI, Myocardial infarction; $L V A D$, left ventricular assist device; $G A P D H$, glyceraldehyde3-phosphate dehydrogenase.

with AMPure XT beads (Beckman Coulter Genomics, Danvers, Mass). Libraries were assessed for concentration and fragment size using a DNA High-Sensitivity Assay on the LabChip GX (PerkinElmer, Waltham, Mass). The libraries were pooled and sequenced on an Illumina HiSeq 2500 system. Data were analyzed using Ingenuity Pathway Analysis (IPA; Ingenuity Systems; www.ingenuity.com).

\section{Statistical Analyses}

All data are presented as mean \pm standard error of the mean. One-way repeated-measures analysis of variance in GraphPad Prism (GraphPad 


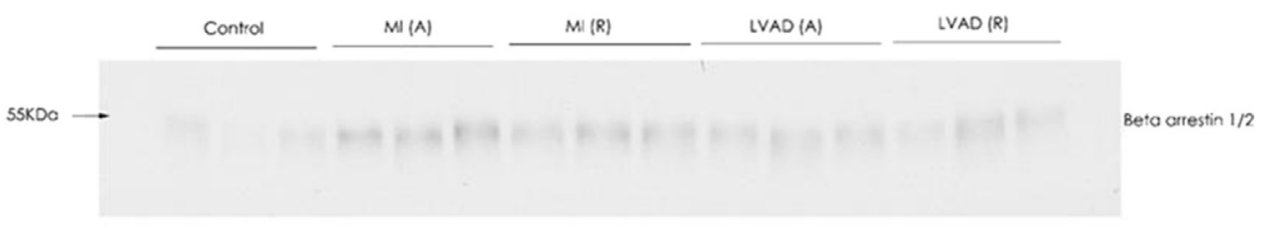

$\beta$-arrestin-bound EGFR

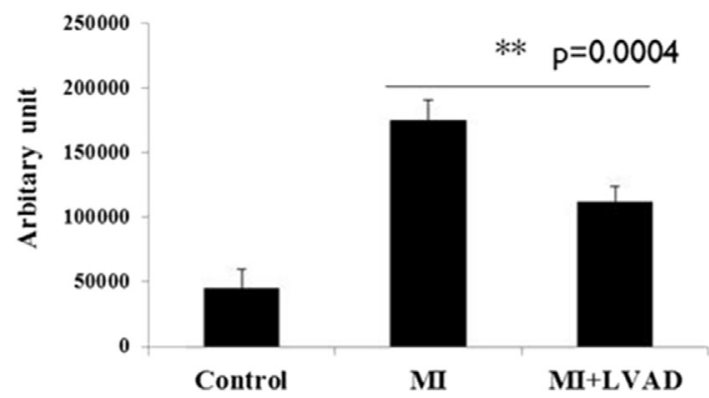

FIGURE 2. Effects of MI and LVAD support on interactions between the EGFR and $\beta$-arrestins. A, adjacent; R, remote. MI increased the amount of EGFR-bound $\beta$-arrestin1/2. Immunoprecipitations and immunoblotting were performed as described in Methods. MI, Myocardial infarction; $L V A D$, left ventricular assist device; $E G F R$, epidermal growth factor receptor.

Software, San Diego, Calif) was used to compare changes in protein expressions on immunoprecipitation and Western blot analysis data. All analyses of variance were followed by multiple comparisons with the least significant difference (Bonferroni) correction. The significance level ( $P$ value) was set at .05.

\section{RESULTS}

\section{Reduces Cytosolic $\beta$-Arrestin Levels, With} Attenuation by LVAD Support

To determine the effects of MI, with or without LVAD support, on $\beta$-arrestin-mediated signaling, we initially examined cytosolic levels of $\beta$-arrestins and posttranslational modification of $\beta$-arrestins by ubiquitination. GPCR signaling through $\beta$-arrestins is known to be inversely related to $\beta$-arrestin levels. Recruitment of $\beta$-arrestins to phosphorylated GPCR tails results not only in recruitment of additional signaling molecules to the complex, but also in $\beta$-arrestin ubiquitination, internalization of the multimolecular complex, and receptor $/ \beta$-arrestin degradation. ${ }^{21}$ Measurement of ubiquitinated proteins may be complicated by their rapid degradation, however. As shown in Figure 1, MI resulted in decreased levels of $\beta$-arrestins, and LVAD support restored normal levels of cytosolic $\beta$-arrestins. Although MI did not result in an increase in detectable ubiquitinated $\beta$-arrestins relative to control specimens, LVAD support resulted in reduced levels of ubiquitinated $\beta$-arrestins, consistent with normalization (ie, increase relative to MI) of overall cytosolic $\beta$-arrestin levels.

\section{Increased EGFR Interaction With $\beta$-Arrestins in} Response to MI, and Suppression by LVAD Support

Cardiovascular mechanical load has previously been shown to activate GPCRs, most notably G $\alpha$ q-coupled receptors. Strain-induced activation of the angiotensin II type 1 receptor $\left(\mathrm{AT}_{1} \mathrm{R}\right)$, which along with the $\beta / \alpha$ adrenergic receptors (ARs) and endothelin $\mathrm{A} / \mathrm{B}$ receptors (ETRs) constitute the most important cardiac GPCRs, results in $\beta$-arrestin 2 activation. $\beta$-arrestin 2 in turn couples the $\mathrm{AT}_{1} \mathrm{R}$ to transactivation of EGFR. ${ }^{22}$ MI resulted in increased interaction between $\beta$-arrestins and EGFR (Figure 2), consistent with increased diastolic myocardial strain regionally and LV end-diastolic volume globally. LVAD support decreased the interaction between $\beta$-arrestins and EGFR, consistent with relatively reduced diastolic myocardial strain and LV end-diastolic volume (Figure 2).

\section{LVAD Support Impairs Activation of Akt}

As discussed previously, numerous studies have suggested that in the heart, chronic $\mathrm{G}$ protein activation is deleterious to cardiomyocyte function and survival. Conversely, $\beta$-arrestinmediated signaling appears to exert beneficial effects on cardiomyocyte function and survival. These effects occur in large part via the kinases Akt and ERK, ${ }^{23}$ which are frequently activated in an apparently adaptive feedback response to various pathological cellular stress stimuli. MI did not induce activation (phosphorylation) of Akt or ERK relative to control levels of Akt/ERK activation (Figure 3); however, MCS via LVAD reduced Akt phosphorylation. Similar results were observed for ERK, although these were not statistically significant. Thus, LV volume unloading via MCS suppresses activation of the cardioprotective effector Akt, and may suppress ERK activation as well.

\section{Regional Effects of MI and Post-MI LVAD Support on Intracellular Signaling Networks}

The aforementioned biochemical studies have suggested that activation of $\beta$-arrestin-mediated signaling in response 

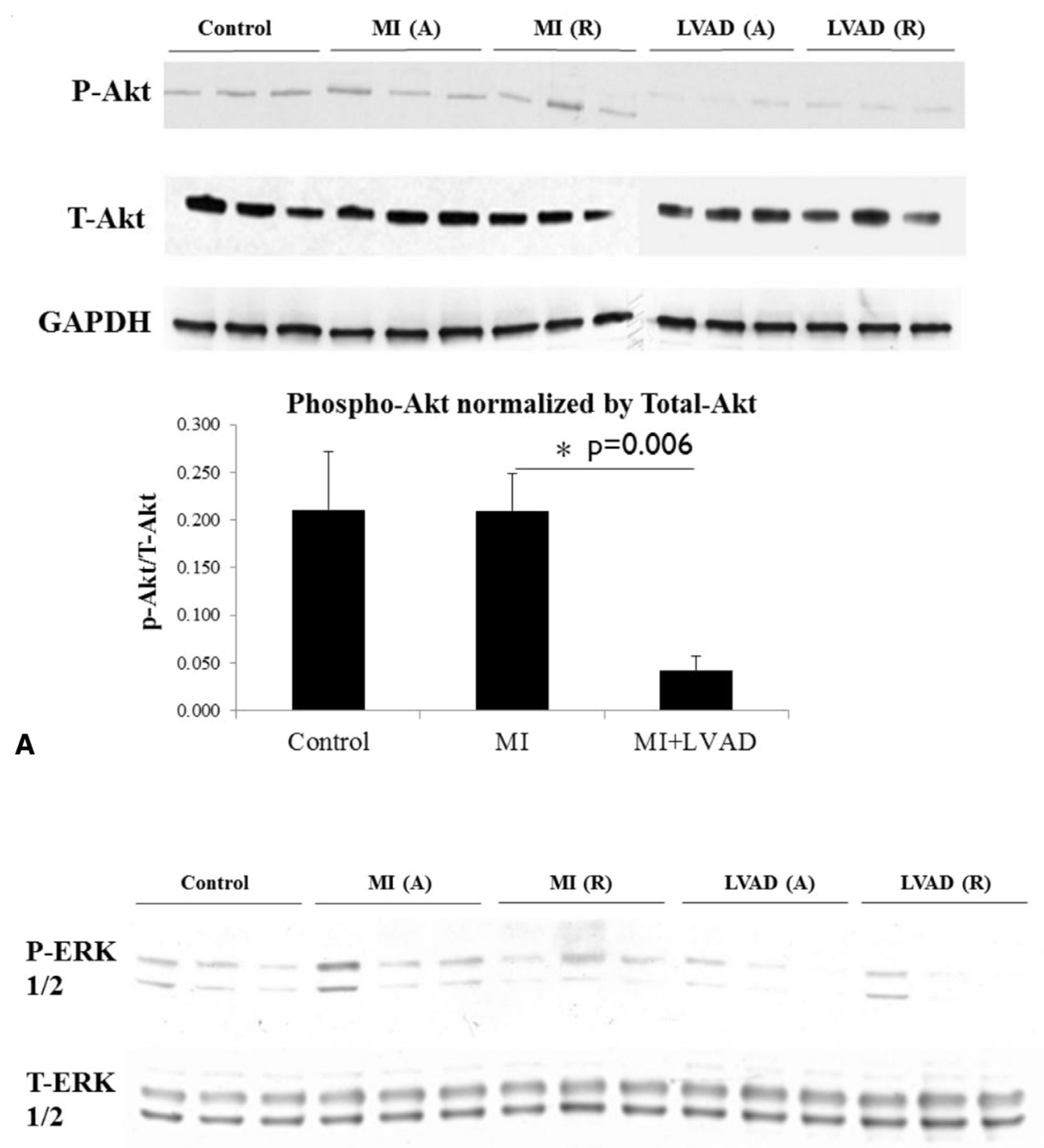

\section{GAPDH}

Phospho-ERK 1/2 normalized by TotalERK1/2

FIGURE 3. Effects of MI and LVAD support on phosphorylation (activation) of Akt and ERK. A, adjacent; R, remote. MI increased the amounts of phospho-Akt and phospho-ERK, whereas LVAD support relatively reduced phospho-Akt and phospho-ERK levels. Immunoblotting was performed as described in Methods. MI, Myocardial infarction; LVAD, left ventricular assist device; $P$-Akt, phospho-Akt; T-Akt, total-Akt, GAPDH, glyceraldehyde3-phosphate dehydrogenase; $P$-ERK 1/2, phospho-extracellular regulated kinase 1/2; T-ERK 1/2, total-extracellular regulated kinase $1 / 2$. 

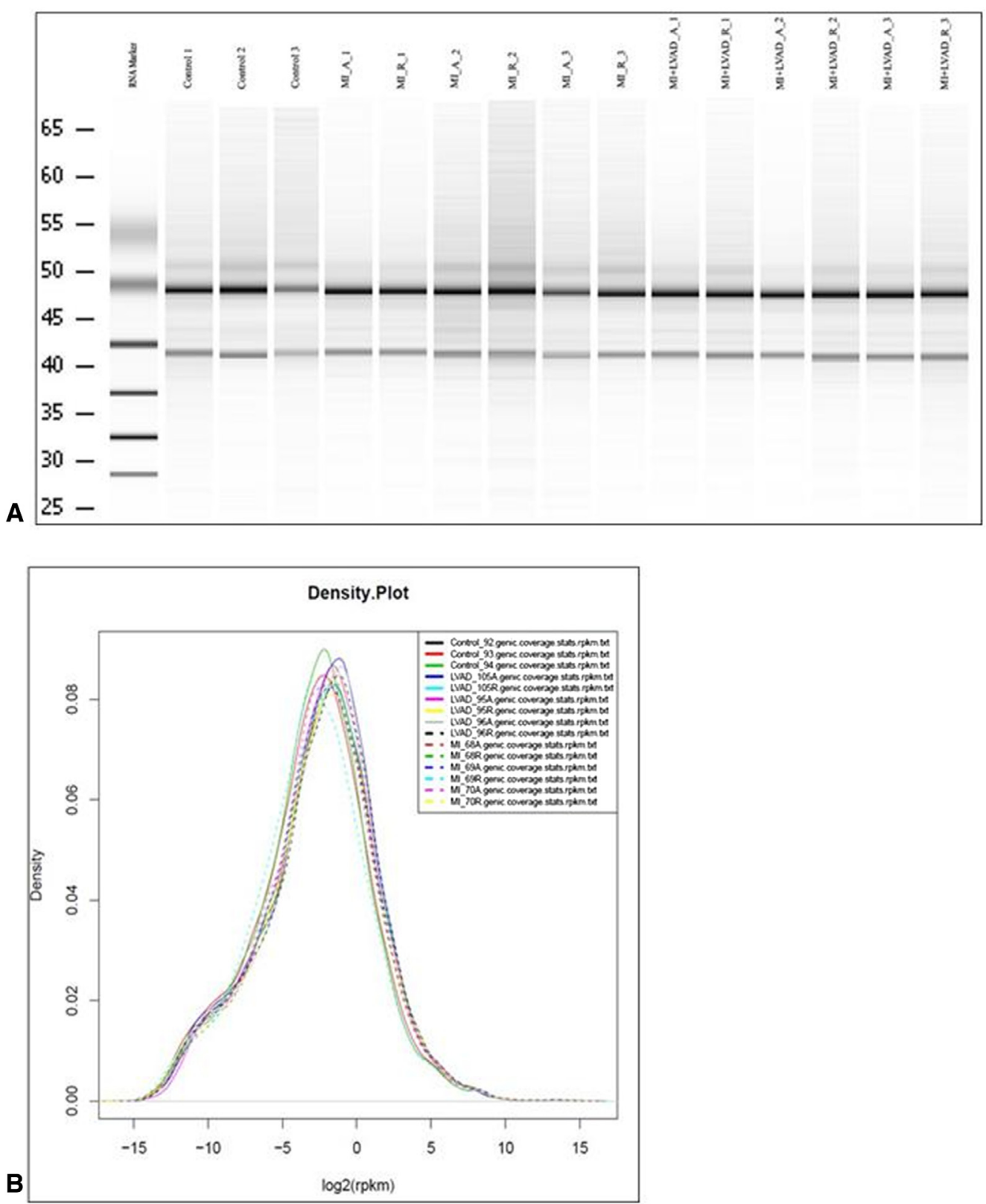

FIGURE 4. Quality of RNA isolation for RNA sequencing studies. RNA isolation was performed as described in Methods. A, Gel electrophoresis characterization of RNA integrity. Numbers denote the numbers of animals. A, adjacent; R, remote. B, RNA density plot demonstrating similar distribution profiles between samples from different animals and tissue regions. MI, Myocardial infarction; LVAD, left ventricular assist device.

to MI is particularly heightened in the MI-adjacent zone, and that LVAD support partially reverses these effects. However, owing to the limited numbers of animal subjects and tissue samples available for analysis, the presence or absence of possible relative differences in $\beta$-arrestinmediated signaling between the MI-adjacent and MI-remote zones could not be determined reliably. RNA sequencing is a highly sensitive approach that can be used to determine large differences in signal transduction pathway activation or suppression based on changes in downstream target gene (mRNA) levels, even with limited sample numbers. ${ }^{24}$ The aims of performing such studies in our experimental system were (1) to identify molecular genetic signatures that could be tightly correlated to specific upstream receptor-driven signal transduction pathways, and (2) to provide more robust signal transduction profiling with limited sample numbers.

RNA samples were prepared from control LV apices, and the MI-adjacent and MI-remote zones of LVs subjected to MI with and without LVAD support. Figure 4 illustrates the quality of RNA isolation from the specimens; RNA quality was found to be homogeneous between the samples. 
TABLE 1. Expression of specific gene transcripts (mRNAs) found to have biologically relevant patterns of expression variation as functions of experimental conditions

\begin{tabular}{lcccc}
\hline Gene & $\begin{array}{c}\text { MI (A) } \\
\text { vs control }\end{array}$ & $\begin{array}{c}\text { MI (A) } \\
\text { vs MI (R) }\end{array}$ & $\begin{array}{c}\text { MI (A) vs } \\
\text { LVAD (A) }\end{array}$ & $\begin{array}{c}\text { LVAD (A) } \\
\text { vs LVAD (R) }\end{array}$ \\
\hline NPPA & 1.779 & 4.674 & 4.408 & - \\
F2RL1 & 3.248 & 2.185 & 2.713 & - \\
SPP1 & - & 1.542 & 2.071 & - \\
MYL4 & - & 3.007 & 1.247 & - \\
MLY9 & - & 1.045 & - & - \\
MYL1 & - & 3.098 & 3.421 & - \\
MYL7 & 1.413 & - & - & - \\
\hline
\end{tabular}

Log fold-change (LFCs) ratios are listed, using base 2 logarithm (mRNA expression ratio of first condition/second condition $=2^{\mathrm{LFC}}$. . NPPA and F2RL1 were increased in the MI (A) zone relative to control samples, and, importantly, the increase in MI (A) was preserved relative to the internally controlled (same animals) MI (R) zones. LVAD support normalized NPPA and F2RL1 expression in the LVAD (A) zone, such that both NPPA and F2RL1 expression was decreased relative to MI (A) and the internally controlled LVAD (R) zone. MI, Myocardial infarction; $A$, adjacent; $R$, remote; $L V A D$, left ventricular assist device.

RNA sequencing was then performed. The following comparisons were of particular biological interest: (1) MI-adjacent zone versus control, (2) MI-adjacent versus MI-remote zone, (3) MI-adjacent zone without or with LVAD support, and (4) MI-adjacent versus MI-remote zone in the setting of LVAD support. Four transcripts were found to vary consistently in a physiologically relevant fashion; the results are displayed in Table 1 mRNA levels for NPPA, the gene that encodes atrial natriuretic peptide, ${ }^{25}$ were substantially increased in the MI-adjacent zone relative to the MI-remote zone (as well as relative to control animal specimens); this constitutes a highly stringent internally controlled difference, in that interanimal heterogeneity cannot explain the finding. LVAD support not only reduced NPPA expression in the MI-adjacent zone relative to the MI-adjacent zone in the absence of LVAD support (ie, between animals subjected to MI alone vs MI plus LVAD support), but also reduced NPPA expression in the MI-adjacent zone relative to the MI-remote zone (ie, internally controlled within the same animal). A nearly identical expression profile was observed for F2RL1, which encodes the protease-activated receptor-2, a GPCR that has been implicated in the regulation of inflammatory signaling and has complex effects on cardiac function. ${ }^{26}$ Differences were also demonstrated in expression profiles for osteopontin $(S P P 1)^{27}$ and several myosin light chain $(M Y L)$ isoforms, but these did not exhibit a tight correlation with the presence or absence of MI with or without LVAD support.

NPPA is induced in response to ventricular and atrial mechanical loading, as well as biochemical stresses. Signal transduction through cardiac GPCRs and non-GPCR growth factor receptors are thought to be the principal regulators of transcription. Specifically, previous studies
TABLE 2. Diastolic strains in MI-adjacent (A) versus MI-remote (R) LV myocardial zones

\begin{tabular}{|c|c|c|c|}
\hline & Pre & 12 weeks & Change \\
\hline \multicolumn{4}{|l|}{ Control } \\
\hline $1 \mathrm{~A}$ & 0 & 7.95 & 7.95 \\
\hline $3 \mathrm{~A}$ & 6.33 & 14.77 & 8.44 \\
\hline Mean \pm SD & $3.17 \pm 4.48$ & $11.36 \pm 4.82$ & $8.20 \pm 0.35$ \\
\hline $1 \mathrm{R}$ & 0.6 & 18.72 & 18.12 \\
\hline $3 \mathrm{R}$ & 2.73 & 17.12 & 14.39 \\
\hline Mean \pm SD & $1.67 \pm 1.51$ & $17.92 \pm 1.13$ & $16.26 \pm 2.64$ \\
\hline \multicolumn{4}{|l|}{ MI } \\
\hline $1 \mathrm{~A}$ & 3.94 & 45.18 & 41.24 \\
\hline $2 \mathrm{~A}$ & 5.39 & 27.21 & 21.82 \\
\hline $3 \mathrm{~A}$ & 0.29 & 50.92 & 50.63 \\
\hline Mean \pm SD & $3.21 \pm 2.63$ & $41.10 \pm 12.37$ & $37.90 \pm 14.69$ \\
\hline $1 \mathrm{R}$ & 5.67 & 26.56 & 20.89 \\
\hline $2 \mathrm{R}$ & 12.69 & 11.27 & -1.42 \\
\hline $3 \mathrm{R}$ & 1.88 & 19.09 & 17.21 \\
\hline Mean \pm SD & $6.75 \pm 5.48$ & $18.97 \pm 7.65$ & $12.22 \pm 11.96$ \\
\hline \multicolumn{4}{|l|}{$\mathrm{MI}+\mathrm{LVAD}$} \\
\hline $1 \mathrm{~A}$ & 11.70 & 33.65 & 21.95 \\
\hline $2 \mathrm{~A}$ & 5.25 & 34.73 & 29.48 \\
\hline $3 \mathrm{~A}$ & 6.55 & 28.21 & 21.66 \\
\hline Mean \pm SD & $7.83 \pm 3.41$ & $32.20 \pm 3.49^{*}$ & $24.36 \pm 4.43^{*}$ \\
\hline $1 \mathrm{R}$ & 3.57 & 15.91 & 12.34 \\
\hline $2 \mathrm{R}$ & 0.75 & 14.46 & 13.71 \\
\hline $3 \mathrm{R}$ & 1.76 & 19.8 & 18.04 \\
\hline Mean \pm SD & $2.03 \pm 1.43$ & $16.72 \pm 2.76$ & $14.70 \pm 2.98$ \\
\hline
\end{tabular}

Sonomicrometry crystals were implanted as described in Methods. Post-left anterior descending artery ligation/pre-LVAD implantation (in the MI and MI + LVAD groups, respectively) and 12-week $\mathrm{A}$ and $\mathrm{R}$ zone diastolic strains are listed, are changes in these values. One control animal (animal 2) did not have interpretable sonomicrometry data. A and R zones for control animals correspond to territories in which MI would have been created. $A$, Adjacent; $S D$, standard deviation; $R$, remote; $M I$, myocardial infarction; $L V A D$, left ventricular assist device. ${ }^{*} P<.05$ for diastolic strains between the A and R zones for MI + LVAD group animals. Because of 1 apparent outlier in the MI group (animal 2), not only were differences between the MI and MI + LVAD group A zone diastolic strains observed, but even intra-animal A versus R zone diastolic strain differences in the MI group were not statistically significant.

have demonstrated that positive (agonist) $G$ proteinmediated signaling through $\mathrm{AT}_{1} \mathrm{R}^{28}{ }^{28} \alpha_{1}-\mathrm{AR},{ }^{29}$ and $\mathrm{ET}_{\mathrm{A}} \mathrm{R}^{30}$ increases NPPA transcription; conversely, positive signaling through the $\beta$-ARs results in decreased NPPA transcription. IPA assessment of the RNA sequencing data found that differential signaling through the $\mathrm{AT}_{1} \mathrm{R}$ and $\alpha_{1}$-AR, but not through the $\beta$-ARs or ETRs, could account for the findings observed. Regulation of F2LRI expression is not well understood; however, IPA results are consistent with those for NPPA, suggesting regulation by $\alpha_{1}$-AR or $\mathrm{AT}_{1} \mathrm{R}$ signaling upstream of gene transcription or posttranscriptional control.

\section{Correlative Effects of LVAD Support on MI-Adjacent Zone Diastolic Strain}

Our previous study ${ }^{5}$ focused on the effects of LVAD support on regional LV remodeling. Specifically, partial 


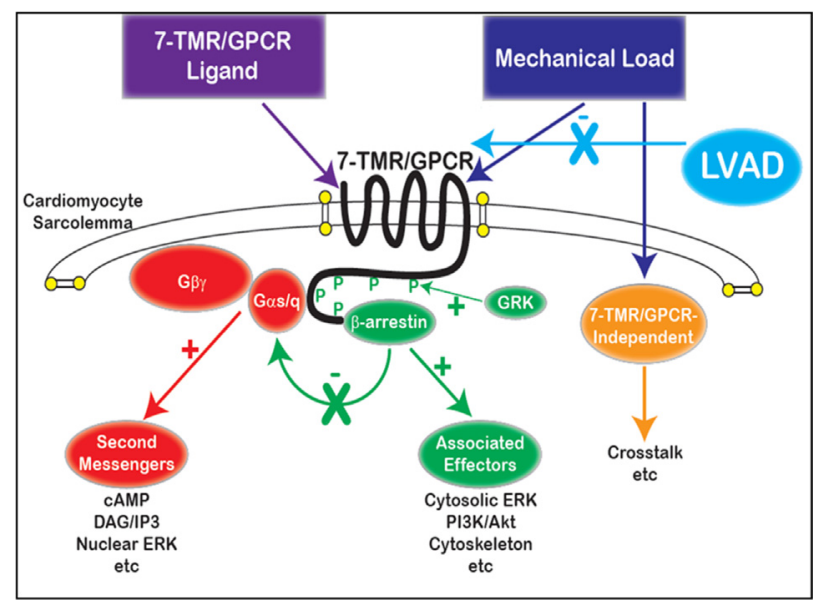

FIGURE 5. Cartoon diagram of the hypothesized effects of mechanical loading, with or without LVAD support, on cardiac 7-TMR/GPCR signal transduction. Mechanical load is depicted as acting like a ligand in facilitating cell surface receptor (GPCR) activation. This activates both $\mathrm{G}$ protein-mediated signaling and $\beta$-arrestin-mediated signaling, the latter of which also suppresses $\mathrm{G}$ protein coupling to the receptor (desensitization). As discussed in the text, chronic $\mathrm{G}$ protein and second messenger activation is cytotoxic, whereas several lines of evidence demonstrate that $\beta$-arrestinmediated signaling is cytoprotective. Thus, it is suggested that load reduction might not selectively reduce $\mathrm{G}$ protein activation, and might reduce $\beta$-arrestin activation. The effects of extent of loading/unloading and timing of LVAD support on differential pathway activity are unclear. TMR, Transmembrane receptor; $G P C R, \mathrm{G}$ protein-coupled receptor; $L V A D$, left ventricular assist device; $G R K$, G protein-coupled receptor kinase; $c A M P, 3^{\prime}, 5^{\prime}$-cyclic adenosine monophosphate; $D A G$, diacylglycerol; $I P 3$, inositol triphosphate; $P 13 K$, phosphatidylinositol-3-kinase; $E R K$, extracellular regulated kinase.

LV unloading with the Impella 5.0 LVAD for only 2 weeks post-MI was found to reduce diastolic strain (ie, local cardiomyocyte stretch/preloading) in the MI-adjacent zone at up to 12 weeks after MI. The signaling analyses performed in this study were from a subset of the aforementioned animals with sufficient specimens to conduct the signal transduction studies. Sonomicrometry measurement-derived regional diastolic strain data from these animals are displayed in Table 2. In brief, no statistically significant differences in MI-adjacent zone diastolic strain were observed with LVAD support between the 3 animals subjected to unsupported MI and the 3 animals that received LVAD support after MI. However, this finding appears to be related to the fact that 1 animal in the MI group had much lower adjacent zone diastolic strain values than the other 2 animals, such that LV diastolic strain was not statistically significantly higher in the adjacent zone versus the remote zone within the MI group animals, which has been demonstrated by previous studies, including ours with a larger number of animals than the subset analyzed. ${ }^{5}$ Moreover, the levels of the $\beta$-arrestin-activated effectors phospho-Akt and phospho-ERK1/2 (Figure 3) are directly proportional to diastolic strain; the second MI group sample that exhibited lower levels of phospho-Akt and phospho-ERK1/2 was from the LV/animal with the lowest diastolic strain in the group. This findings is consistent with mechanical load-induced stimulation of $\beta$-arrestinmediated signal transduction.

\section{DISCUSSION}

In this study, we used a post-MI model of LV systolic dysfunction and investigated the effects of LVAD support on (1) $\beta$-arrestin-mediated signaling and (2) activity of signaling networks as a function of myocardial region. We found that LVAD support suppressed cardioprotective $\beta$-arrestin-mediated signal transduction; however, this appeared to occur in the overall context of normalization of signaling networks in the LVs of LVAD-supported hearts, particularly in the MI-adjacent zones (border zones) of myocardium. These findings suggest that although LV MCS has beneficial effects on myocardial signal transduction as a whole, which translates to improved calcium handling and cardiomyocyte function, cytoprotective signaling may be suppressed in addition to cytotoxic signaling.

MCS for chronic LV failure is now a well-established treatment with validated benefits over HF pharmacotherapy alone. In contrast, short-term MCS is comparatively less well accepted in the treatment of acute HF/cardiogenic shock, although recent evidence and even consensus documents support more widespread application. LVAD support as a mode of therapy to actually prevent LV pathological remodeling and enhance LV function and recovery is even more controversial. Our group's studies, as well as those of others, suggest that this novel application of MCS is reasonable and effective; yet MCS carries an inherent procedural risk, and its benefits may be further limited. Pathological stimuli exert directly deleterious effects through pathologically activated or suppressed signaling pathways, but also induce adaptive cytoprotective signals. In MI-induced LV dysfunction, pathological loading conditions are present regionally in the MI-adjacent zone. LVAD support normalizes loading conditions in the adjacent zone, and although this suppresses $\mathrm{G} \alpha \mathrm{q}$-mediated $\alpha_{1}$-AR/AT $\mathrm{A}_{1} \mathrm{R}$ signaling, it also suppresses $\beta$-arrestin-mediated signaling (Figure 5).

It is possible that the differential effects on each pathway may be related to changes in the extent and duration of mechanical unloading, and that an optimal point for extent and/or duration of LVAD support exists; however, it is also possible that suppression of cytoprotective signaling is an unavoidable side effect of inhibiting cytotoxic $\mathrm{G} \alpha \mathrm{q}-$ mediated signaling. Adjunctive medical therapies, such as selective GPCR ligands ("biased agonists") that only activate $\beta$-arrestin-mediated signaling, may have utility in treating $\mathrm{LV}$ dysfunction. One such ligand for $\mathrm{AT}_{1} \mathrm{R}$ is currently in clinical trials for use in acutely decompensated HF. 
This study has some important methodological limitations. First, the MI model used did not incorporate reperfusion, which is normally performed when feasible. Thus, the results should be interpreted in the context of completed large-territory MI without revascularization, which is a minority of MIs. Second, standard HF pharmacotherapies were not used; however, these therapies, such as $\beta$-AR antagonists and angiotensin-converting enzyme inhibitors or $\mathrm{AT}_{1} \mathrm{R}$ antagonists, themselves nonselectively suppress both $\mathrm{G}$ protein- and $\beta$-arrestin-mediated signaling, not substantially different from those of LVAD support alone. Moreover, the roles of standard HF pharmacotherapies in the setting of LVAD support have not been validated. Third, the model as developed is one of LV dysfunction and not necessarily of clinical HF; we did not ascertain whether the end-organ features of HF are present in the model. Finally, the initial biochemical studies are limited by sample numbers, which was one reason why we used the statistically robust RNA sequencing approach for subsequent analyses.

In conclusion, these studies demonstrate that small platform catheter-based LVAD support exerts suppressive effects on cardioprotective $\beta$-arrestin-mediated signal transduction, while normalizing the signaling networks of $\mathrm{G} \alpha \mathrm{q}$-coupled cardiac GPCRs in the MI-adjacent zone. Further studies are needed to elucidate the roles of specific GPCRs in directed $\beta$-arrestin-mediated signaling in post-MI LV dysfunction, and to define the potential optimal titration of LVAD support based on signaling and genetic markers in addition to standard LV functional endpoints.

\section{Conflict of Interest Statement}

Authors have nothing to disclose with regard to commercial support.

You can watch a Webcast of this AATS meeting presentation by going to: http://webcast.aats.org/2015/Video/ Tuesday/04-28-15_6B_0750_Rajagopal.mp4

The authors thank Sean Daugherty and Amol Shetty of the University of Maryland Institute of Genome Sciences for processing Ingenuity IPA pathway analyses.

\section{References}

1. Hellermann JP, Goraya TY, Jacobsen SJ, Weston SA, Reeder GS, Gersh BJ, et al. Incidence of heart failure after myocardial infarction: is it changing over time? Am J Epidemiol. 2003;157:1101-7.

2. Reynolds HR, Hochman JS. Cardiogenic shock: current concepts and improving outcomes. Circulation. 2008;117:686-97.

3. Slaughter MS, Rogers JG, Milano CA, Russell SD, Conte JV, Feldman D, et al. Advanced heart failure treated with continuous-flow left ventricular assist device. N Engl J Med. 2009;361:2241-51.

4. Aaronson KD, Slaughter MS, Miller LW, McGee EC, Cotts WG, Acker MA, et al. Use of an intrapericardial, continuous-flow, centrifugal pump in patients awaiting heart transplantation. Circulation. 2012;125:3191-200.

5. Wei X, Li T, Hagen B, Zhang P, Sanchez PG, Williams K, et al. Short-term mechanical unloading with left ventricular assist devices after acute myocardial infarction conserves calcium cycling and improves heart function. JACC Cardiovasc Interv. 2013;6:406-15.

6. Blom AS, Pilla JJ, Arkles J, Dougherty L, Ryan LP, Gorman JH III, et al. Ventricular restraint prevents infarct expansion and improves border zone function after myocardial infarction: a study using magnetic resonance imaging, three-dimensional surface modeling, and myocardial tagging. Ann Thorac Surg. 2007;84:2004-10.

7. de Tombe PP. Cardiac myofilaments: mechanics and regulation. J Biomech. 2003;36:721-30.

8. Tibayan FA, Lai DT, Timek TA, Dagum P, Liang D, Daughters GT, et al. Alterations in left ventricular torsion in tachycardia-induced dilated cardiomyopathy. J Thorac Cardiovasc Surg. 2002;124:43-9.

9. Yankey GK, Li T, Kilic A, Cheng G, Satpute A, Savai K, et al. Regional remodeling strain and its association with myocardial apoptosis after myocardial infarction in an ovine model. J Thorac Cardiovasc Surg. 2008;135:991-8.

10. Rajagopal K, Lefkowitz RJ, Rockman HA. When 7 transmembrane receptors are not G protein-coupled receptors. J Clin Invest. 2005;115:2971-4.

11. Rajagopal S, Rajagopal K, Lefkowitz RJ. Teaching old receptors new tricks: biasing seven-transmembrane receptors. Nat Rev Drug Discov. 2010;9:373-86.

12. Zou Y, Akazawa H, Qin Y, Sano M, Takano H, Minamino T, et al. Mechanical stress activates angiotensin II type 1 receptor without the involvement of angiotensin II. Nat Cell Biol. 2004;6:499-506.

13. Chachisvilis M, Zhang YL, Frangos JA. G protein-coupled receptors sense fluid shear stress in endothelial cells. Proc Natl Acad Sci U S A. 2006;103:15463-8.

14. Scimia MC, Hurtado C, Ray S, Metzler S, Wei K, Wang J, et al. APJ acts as a dual receptor in cardiac hypertrophy. Nature. 2012;488:394-8.

15. Woodall MC, Ciccarelli M, Woodall BP, Koch WJ. G protein-coupled receptor kinase 2: a link between myocardial contractile function and cardiac metabolism. Circ Res. 2014;114:1661-70.

16. Lefkowitz RJ, Rajagopal K, Whalen EJ. New roles for beta-arrestins in cell signaling: not just for seven-transmembrane receptors. Mol Cell. 2006;24: 643-52.

17. Rajagopal K, Whalen EJ, Violin JD, Stiber JA, Rosenberg PB, Premont RT, et al. Beta-arrestin2-mediated inotropic effects of the angiotensin II type 1A receptor in isolated cardiac myocytes. Proc Natl Acad Sci U S A. 2006;103:16284-9.

18. Kim KS, Abraham D, Williams B, Violin JD, Mao L, Rockman HA. $\beta$-arrestinbiased AT1R stimulation promotes cell survival during acute cardiac injury. Am J Physiol Heart Circ Physiol. 2012;303:H1001-10.

19. Klotz S, Barbone A, Reiken S, Holmes JW, Naka Y, Oz MC, et al. Left ventricular assist device support normalizes left and right ventricular beta-adrenergic pathway properties. J Am Coll Cardiol. 2005;45:668-76.

20. Hata JA, Williams ML, Schroder JN, Lima B, Keys JR, Blaxall BC, et al. Lymphocyte levels of GRK2 (betaARK1) mirror changes in the LVAD-supported failing human heart: lower GRK2 associated with improved beta-adrenergic signaling after mechanical unloading. J Card Fail. 2006;12:360-8.

21. Shenoy SK, McDonald PH, Kohout TA, Lefkowitz RJ. Regulation of receptor fate by ubiquitination of activated beta 2-adrenergic receptor and beta-arrestin. Science. 2001;294:1307-13.

22. Kim J, Ahn S, Rajagopal K, Lefkowitz RJ. Independent beta-arrestin2 and Gq/protein kinase Czeta pathways for ERK stimulated by angiotensin type 1A receptors in vascular smooth muscle cells converge on transactivation of the epidermal growth factor receptor. J Biol Chem. 2009;284:11953-62.

23. Rakesh K, Yoo B, Kim IM, Salazar N, Kim KS, Rockman HA Beta-arrestin-biased agonism of the angiotensin receptor induced by mechanical stress. Sci Signal. 2010;3:ra46.

24. Yang KC, Yamada KA, Patel AY, Topkara VK, George I, Cheema FH, et al. Deep RNA sequencing reveals dynamic regulation of myocardial noncoding RNAs in failing human heart and remodeling with mechanical circulatory support. Circulation. 2014;129:1009-21.

25. Houweling AC, van Borren MM, Moorman AF, Christoffels VM. Expression and regulation of the atrial natriuretic factor encoding gene Nppa during development and disease. Cardiovasc Res. 2005;67:583-93.

26. Antoniak S, Pawlinski R, Mackman N. Protease-activated receptors and myocardial infarction. IUBMB Life. 2011;63:383-9.

27. Klingel K, Kandolf R. Osteopontin: a biomarker to predict the outcome of inflammatory heart disease. Semin Thromb Hemost. 2010;36:195-202.

28. Majalahti T, Suo-Palosaari M, Sármán B, Hautala N, Pikkarainen S, Tokola H, et al. Cardiac BNP gene activation by angiotensin II in vivo. Mol Cell Endocrinol. 2007:273:59-67.

29. Knowlton KU, Baracchini E, Ross RS, Harris AN, Henderson SA, Evans SM, et al. Co-regulation of the atrial natriuretic factor and cardiac myosin light 
chain-2 genes during alpha-adrenergic stimulation of neonatal rat ventricular cells: identification of cis sequences within an embryonic and a constitutive contractile protein gene which mediate inducible expression. J Biol Chem. 1991;266: 7759-68.

30. Shubeita HE, McDonough PM, Harris AN, Knowlton KU, Glembotski CC, Brown JH, et al. Endothelin induction of inositol phospholipid hydrolysis, sarcomere assembly, and cardiac gene expression in ventricular myocytes: a paracrine mechanism for myocardial cell hypertrophy. J Biol Chem. 1990;265: 20555-62.

Key Words: myocardial infarction, left ventricular dysfunction, mechanical circulatory support, mechanotransduction, intracellular signaling

\section{Discussion}

Dr Mark Slaughter (Louisville, Ky). I'd like to thank Dr Rajagopal for an excellent presentation and the continued work to try and determine if partial support from a catheter-based ventricular assist device improves cardiac function after an acute myocardial infarction.

I have four questions for the author.

In the previous work from the Maryland group, there was detailed information from the sonomicrometry crystal arrays as well as the degree of support. It would be helpful to know regional wall thickness and volumes. Are we to assume that this is identical from the previous study, and how did it compare to your previous work?

Dr Rajagopal. These were actually animals that were done in the previous studies. These were biochemical analyses that were not performed previously that now have been done. I did not go back to correlate what the biochemical findings were in relation to proportionality to regional differences at the time, nor have we, but it's something that is certainly worth doing. But these are those identical animals.

Dr Slaughter. So in that case, though, only half the animals were actually studied in this section. Why weren't all the animals included?

Dr Rajagopal. That is an excellent question and just simply due to lack of tissue availability for all of the specimens. It was just a practical limitation.

Dr Slaughter. We also have an interest in partial support strategies and have demonstrated that partial support will move the PV loop slightly to the left but also adversely affects coronary blood flow, as demonstrated by microsphere studies. What role do you think inadequate coronary artery perfusion has on your results?

Similarly, as you noted in the limitations, there was no reperfusion strategy. Would this limit the adverse findings and negate the need for a partial support pump?

Dr Rajagopal. I think that is a very fair concern and criticism. Certainly the lack of reperfusion limits practical applicability. That said, there is yet a substantial subset of patients clinically who do not achieve reperfusion or it is inefficacious or even too late. But that I think is an established limitation of a ligation model, the lack of reperfusion, and I recognize that there is not a ready way to get around that.

With regard to the effect, Dr Slaughter, of partial support on coronary blood flow, we did not examine that in this study. I can hypothesize with respect to an anticipated result, but I can't give you data in regards to that.

Dr Slaughter. In chronic heart failure, gene triggers essentially will start to normalize at around 12 to 18 months. So the normalization of the signaling network could merely represent that at 12 weeks the acute injury is over, so signaling has returned to baseline. However, it seems as though the real goal of the partial support pump was to maintain the $\beta$-arrestin signaling, which is cardioprotective, and it did not. So what do you really hypothesize is the mechanism for the improved results that you see?

Dr Rajagopal. Because, as I discussed earlier in one of the model slides, load is a proximal stimulus. The way load activates GPCR, we think at least, that it acts as if it were a ligand. and so, yes, it may suppress cardioprotective $\beta$-arrestin-mediated signaling, but what it is really doing is getting rid of toxic $G$ protein-mediated signaling. And that has been well established. There are experimental and animal studies to demonstrate that.

I suppose the way I would interpret these data are that not everything is good about a VAD, which is to say, yes, it may get rid of pathological loading and pathological signaling, but it may also, if you will, throw the baby out with the bathwater and get rid of adaptive responses to the pathological stimuli.

Dr Slaughter. Agree. So the idea of partial support becomes very important in regards to how much you unload the heart.

Final question: The catheter-based device was left in place for 2 weeks. This is not clinically practical. How do you think these results would be affected with only 24 to 48 hours of support?

Thank you for the opportunity to read the manuscript and discuss your presentation.

Dr Rajagopal. Thank you, Dr Slaughter. Again, that is an excellent question and one of great clinical relevance. Two weeks certainly is not clinically practical; however, people certainly have used catheter-based VADs for that duration of time, albeit off-label.

With regard to duration, ideally I think, because most patients do get reperfused, the duration of required support I would hypothesize to be less because there is a limitation in infarct size that occurs as a consequence of reperfusion. 